\title{
Observations of Cepheid Variables in Two LMC Clusters Using EROS 2 Data
}

\author{
V. Ripepi \\ Osservatorio Astronomico di Capodimonte (Napoli), Italy
}

F. Bauer

College de France, Paris, France

J.P. Beaulieu

Institut d'Astrophysique de Paris, France

\section{Introduction}

It is well known that Cepheid variables are very important distance indicators because of their PL and PLC relations. The distances derived through the quoted relations are affected by well known systematic effects, since, in general, we have a poor knowledge of both reddening and metallicity of the Cepheid sample. Good estimates of the quoted parameters can be achieved if the observed Cepheids are cluster members since, by comparison between cluster CMD and theoretical isochrones we can estimate the cluster reddening, metallicity and age, i.e., the evolving masses for each evolutionary phase. The ideal objects to be searched for Cepheid membership are Magellanic Cloud clusters since they are well populated in the relevant evolutionary phases.

The EROS 2 project (see Bauer et al. 1999, and other contributions in this book), among the other important results, provided excellent light curves and relevant parameters for Cepheid variables in the field of the two LMC cluster NGC 1943 and NGC 1958. Since the EROS 2 photometric system is difficult to calibrate, we coupled EROS 2 observations for Cepheids with new observations carried out in the standard $B V R_{J} I_{J}$ filter system, in order to derive accurate CMDs for comparison with theoretical models. In this contribution we present some preliminary results of this work.

\section{Observations and Preliminary Results}

The observations were carried out partly in November 1996 with the $0.90 \mathrm{~m}$. "Dutch" Telescope (for NGC 1958) and partly with the $1.5 \mathrm{~m}$ "Danish" during 1998, January, both at ESO (La Silla). Observations with the "Dutch" telescope consist of 11 observing nights spaced along 21 days, so they allow a search for stellar variability. A detailed report about reduction and calibration procedure will be done in a forthcoming paper.

Concerning some preliminary results, there are ten variable stars both in NGC 1943 and in NGC 1958. Most of these stars are Cepheid already present 

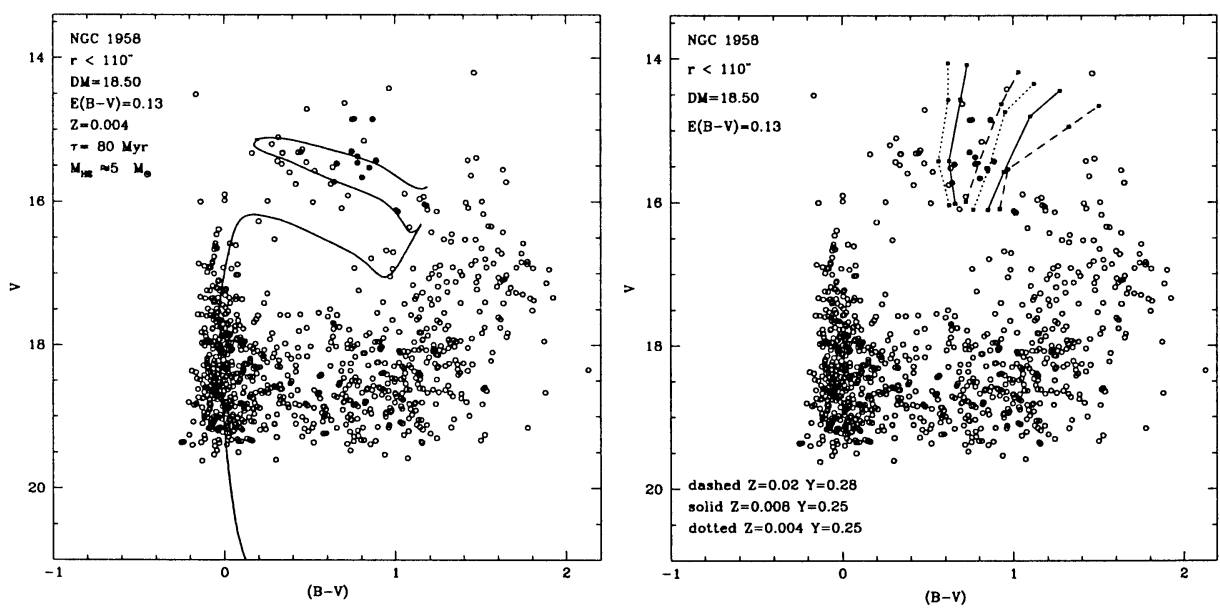

Figure 1. Left panel: C-M diagram for NGC 1958; filled circles represent variable stars, whereas the solid line shows the theoretical isochrone for the labelled values of age and metallicity; Right panel: as before, but the various lines represents the instability strips for various metallicities by Bono, Marconi \& Stellingwerf (1999).

in the EROS 2 database. From the comparison between observed CMDs and theoretical isochrones (with the new input physics by Degl'Innocenti \& Marconi 1998) we estimated the cluster metallicity, reddening and age as well as the Cepheid evolving masses. In Fig. 1 (left) we report the result of such a procedure for NGC 1958, but the same analysis was applied to NGC 1943. We note that adopting a set of isochrones with different input physics (e.g. Bertelli et al. 1994), the data are fitted equally well with the following values for the relevant parameters: $\mathrm{Z}=0.008, \mathrm{DM}=18.30, E(B-V)=0.13$, Age $=100-125$ Myrs. This occurrence, which would deserve a more detailed discussion, can be interpreted as a measure of the uncertainties still affecting the intermediate mass star evolutionary models. Finally we compare in Fig. 1 (right) the observed instability strip for NGC 1958 with the theoretical one (Bono, Marconi \& Stellingwerf, 1999) for various assumptions about chemical composition. From the figure we notice that, at least qualitatively, the agreement between the theory of pulsations and the observations seems satisfactory.

Acknowledgments. We wish to thank Dr. Degl'Innocenti and Dr. Marconi for providing their unpublished isochrones. We thank also Dr. Bono and Dr. Marconi for providing theoretical results in advance of publication.

\section{References}

Bauer, et al. (the EROS collaboration) 1999, A\&A, 348, 175

Bertelli, G., et al. 1994, A\&AS, 106, 275

Bono, G., Marconi, M., \& Stellingwerf, R. 1999, ApJS, 122, 167

Degl'Innocenti S, Marconi, M. 1998 (private communication) 